UDC 517.53

S. L. WALI

\title{
AN INTEGRAL ESTIMATE FOR AN OPERATOR PRESERVING INEQUALITIES BETWEEN POLYNOMIALS
}

\begin{abstract}
Let $\mathcal{P}_{n}$ be the class of polynomials of degree at most $n$. Rahman introduced a class $\mathcal{B}_{n}$ of operators $B$ that map $\mathcal{P}_{n}$ into itself. In this paper we prove some results concerning the integral estimates of such operators and, thereby, obtain generalizations, as well as improvements, of some well known $L_{p}$ inequalities.
\end{abstract}

Key words: Polynomials, B-Operator, Inequalities in the complex domain, Zeros.

2010 Mathematical Subject Classification: 30A06, 30A64,30E10

1. Introduction. Let $\mathcal{P}_{n}$ be the class of polynomials $P(z):=\sum_{j=0}^{n} a_{j} z^{j}$ of degree at most $n$ with complex coefficients, and $P^{\prime}(z)$ be its derivative. Let $B$ be a linear operator mapping polynomials from $\mathcal{P}_{n}$ to polynomials in $\mathcal{P}_{n}$. We say that $B$ is a $\mathcal{B}_{n}$-operator if for every polynomial $P$ of degree $n$ having all its zeros in the closed unit disk, $B[P]$ has all its zeros in the closed unit disk. Rahman [10] (see also Rahman and Schmeisser [11, Lemma 14.5.7]) introduced this class $\mathcal{B}_{n}$ of operators $B$ and observed that if $\lambda_{0}, \lambda_{1}$, and $\lambda_{2}$ are such that

$$
\lambda_{0}+C(n, 1) \lambda_{1} z+C(n, 2) \lambda_{2} z^{2} \neq 0, \quad C(n, r):=\frac{n !}{r !(n-r) !}
$$

for $\operatorname{Re}(z) \geqslant \frac{n}{4}$, then the operator $B$, which maps a polynomial $P$ of degree at most $n$ to the polynomial

$$
B[P](z):=\lambda_{0} P(z)+\lambda_{1}\left(\frac{n z}{2}\right) \frac{P^{\prime}(z)}{1 !}+\lambda_{2}\left(\frac{n z}{2}\right)^{2} \frac{P^{\prime \prime}(z)}{2 !}
$$

(C) Petrozavodsk State University, 2020 
is a $\mathcal{B}_{n}$-operator. This can also be deduced from a result of Marden [9, Corollary 18.3]. Concerning this class of operators, Rahman [10] observed:

$$
|B[P]| \leqslant M\left|B\left[E_{n}\right]\right|, \quad|z| \geqslant 1,
$$

whenever

$$
|P| \leqslant M, \quad|z|=1,
$$

where $|B[P]|=|B[P(z)]|$ and $E_{n}(z)=z^{n}$.

The famous Bernstein inequality [5] is: for every $P \in \mathcal{P}_{n}$

$$
\max _{|z|=1}\left|P^{\prime}(z)\right| \leqslant n \max _{|z|=1}|P(z)| ;
$$

it is a special case of Inequality (1) and follows from it with a suitable choice of $\lambda_{i}, i=0,1,2$.

As an improvement of Inequality (1), Shah and Liman [14] proved:

Theorem A. If $P \in \mathcal{P}_{n}$ and $P(z) \neq 0$ in $|z|<1$, then, for $|z| \geqslant 1$,

$$
|B[P]| \leqslant \frac{1}{2}\left[\left\{\left|B\left[E_{n}\right]\right|+\left|\lambda_{0}\right|\right\} \max _{|z|=1}|P(z)|-\left\{\left|B\left[E_{n}\right]\right|-\left|\lambda_{0}\right|\right\} \min _{|z|=1}|P(z)|\right],
$$

where $E_{n}(z):=z^{n}$.

The result of Erdös-Lax [8], Ankeny-Rivlin [2], and Aziz-Dawood [3] follows from Inequality (3) with a suitable choice of $\lambda_{i}, i=0,1,2$ (see for reference [14]). For every $f \in \mathcal{P}_{n}$, from now onwards we write:

$L_{p}$ norm of $f=\|f\|_{p}=\left\{\frac{1}{2 \pi} \int_{0}^{2 \pi}\left|f\left(e^{i \theta}\right)\right|^{p} d \theta\right\}^{\frac{1}{p}}, 1 \leqslant p<\infty$, whereas $L_{p}$ mean norm of $f=\|f\|_{p}^{*}=\left\{\frac{1}{2 \pi} \int_{0}^{2 \pi}\left|f\left(e^{i \theta}\right)\right|^{p} d \theta\right\}^{\frac{1}{p}}, 0<p<\infty$, and $\|f\|_{\infty}=\max _{|z|=1}|f(z)|$.

Concerning the integral mean inequalities for polynomials, there exists several results proved recently. In this direction, the interested readers may see [16], [13]. In particular, in connection to $L_{p}$ norm of a B-operator, Shah and Liman [15] (see also [17]) proved the following:

Theorem B. If $P \in \mathcal{P}_{n}$ be such that $P(z) \neq 0$ in $|z|<1$, then for every $R \geqslant 1$ and $p \geqslant 1$,

$$
\|B[P](R .)\|_{p} \leqslant \frac{\left|B\left[E_{n}\right](R .)\right|+\left|\lambda_{0}\right|}{\left\|1+E_{n}\right\|_{p}}\|P\|_{p}, \quad|z|=1,
$$


where $B \in \mathcal{B}_{n}$ and $E_{n}(z):=z^{n}$.

Recently Rather and Shah [12] obtained the following result and showed that Theorem $\mathrm{B}$ is valid for $0<p<1$ as well.

Theorem C. Let $P \in \mathcal{P}_{n}$ and $P(z) \neq 0$ in $|z|<1$, then for every $R \geqslant 1,0 \leqslant p<\infty$,

$$
\|B[P](R .)\|_{p}^{*} \leqslant \frac{\left\|R^{n} \wedge z+\lambda_{0}\right\|_{p}^{*}}{\|1+z\|_{p}^{*}}\|P\|_{p}^{*},
$$

where $B \in \mathcal{B}_{n}$ and $\wedge:=\lambda_{0}+\lambda_{1} \frac{n^{2}}{2}+\lambda_{2} \frac{n^{3}(n-1)}{8}$.

In this paper, we extend Theorem A to the $L_{p}$ mean norm and, thereby, obtain a refinement of Theorem C. In fact, we prove:

Theorem 1. If $P \in \mathcal{P}_{n}$ and $P(z)$ does not vanish in $|z|<1$, then, for every $\beta \in \mathcal{C}$ with $|\beta| \leqslant 1, R>1$, and $0 \leqslant p<\infty$,

$$
\left\|B[P](R .)+\left(\frac{R^{n} \wedge z^{n}-\left|\lambda_{0}\right|}{2}\right) m|\beta|\right\|_{p}^{*} \leqslant \frac{\left\|R^{n} \wedge z^{n}+\lambda_{0}\right\|_{p}^{*}}{\|1+z\|_{p}^{*}}\|P\|_{p}^{*},
$$

where

$$
B \in \mathcal{B}_{n}, m:=\min _{|z|=1}|P(z)| \quad \text { and } \quad \wedge:=\lambda_{0}+\lambda_{1} \frac{n^{2}}{2}+\lambda_{2} \frac{n^{3}(n-1)}{8} .
$$

The result is sharp and the extremal polynomial is $P(z)=z^{n}+1$.

If we make $p \rightarrow \infty$ in (5), we get the following sharp result:

Corollary 1. If $P(z)$ is a polynomial of degree $n$, which does not vanish in $|z|<1$, then, for $|z|=1$,

$$
\|B[P](R .)\|_{\infty} \leqslant \frac{1}{2}\left\{\left(R^{n}|\wedge|+\left|\lambda_{0}\right|\right)\|P(z)\|_{\infty}-\left(R^{n}|\wedge|-\left|\lambda_{0}\right|\right) m\right\},
$$

where $B, \wedge$, and $m$ are defined in (6).

The following result, which is an improvement of Theorem B, follows from Theorem 1 by using the triangle inequality.

Corollary 2. If $P \in \mathcal{P}_{n}$ and $P(z)$ does not vanish in $|z|<1$, then for every $\beta \in \mathcal{C}$ with $|\beta| \leqslant 1, \quad R>1$ and $0 \leqslant p<\infty$,

$$
\left\|B[P](R .)+\left(\frac{R^{n} \wedge z^{n}-\left|\lambda_{0}\right|}{2}\right) m|\beta|\right\|_{p}^{*} \leqslant \frac{R^{n}|\wedge|+\left|\lambda_{0}\right|}{\|1+z\|_{p}^{*}}\|P\|_{p}^{*},
$$


where $B \in \mathcal{B}_{n}, \wedge$ and $m$ are defined in (6).

The result is sharp and the equality holds for the polynomial $P(z)=$ $=z^{n}+1$.

Corollary 2 also shows that Theorem $\mathrm{A}$ is valid for $0 \leqslant p<1$ as well. Substituting for $B[P](z)$ and assuming that $\lambda_{i}, i=0,1,2$, are same for all polynomials $P(z)$, we obtain from inequality (5):

$$
\begin{gathered}
\|\left\{\lambda_{0} P(R z)+\lambda_{1}\left(\frac{n z}{2}\right) R \frac{P^{\prime}(R z)}{1 !}+\lambda_{2}\left(\frac{n z}{2}\right)^{2} R^{2} \frac{P^{\prime \prime}(R z)}{2}\right\}+ \\
+\frac{1}{2}\left\{\left(\lambda_{0}+\lambda_{1} \frac{n^{2}}{2}+\lambda_{2} \frac{n^{3}(n-1)}{8}\right) R^{n} z^{n}-\left|\lambda_{0}\right|\right\} m|\beta| \|_{p}^{*} \leqslant \\
\leqslant \frac{\left\|\left(\lambda_{0}+\lambda_{1} \frac{n^{2}}{2}+\lambda_{2} \frac{n^{3}(n-1)}{8}\right) R^{n} z^{n}+\lambda_{0}\right\|_{p}^{*}}{\|1+z\|_{p}^{*}}\|P\|_{p}^{*} .
\end{gathered}
$$

Suppose that $\lambda_{1}=0=\lambda_{2}$, which is possible, as in this case it is easy to see that

$$
\lambda_{0}+C(n, 1) \lambda_{1} z+C(n, 2) \lambda_{2} z^{2} \neq 0 \quad \text { for } \quad \operatorname{Re}(z) \geqslant \frac{n}{4} .
$$

We get from (9) the following refinement of a result earlier proved by Boas and Rahman [6] for $p \geqslant 1$ and Rahman and Schemessier [11] for $0 \leqslant p<1$.

Corollary 3. If $P \in \mathcal{P}_{n}$ and $P(z)$ does not vanish in $|z|<1$, then for every $\beta \in \mathcal{C}$ with $|\beta| \leqslant 1, R>1$ and $0 \leqslant p<\infty$,

$$
\left\|P(R z)+\frac{R^{n} z^{n}-1}{2} m|\beta|\right\|_{p}^{*} \leqslant \frac{\left\|R^{n} z^{n}+1\right\|_{p}^{*}}{\|z+1\|_{p}^{*}}\|P\|_{p}^{*},
$$

where $m=\min _{|z|=1}|P(z)|$.

Again, assuming that $\lambda_{0}=\lambda_{2}=0$, which is also possible, we get the following refinement of de Brujin's theorem [7] for $p \geqslant 1$ and a result of Arestov [1] for $0 \leqslant p \leqslant \infty$.

Corollary 4. If $P \in \mathcal{P}_{n}$ and $P(z)$ does not vanish in $|z|<1$, then for every $\beta \in \mathcal{C}$ with $|\beta| \leqslant 1, R>1$ and $0 \leqslant p<\infty$,

$$
\left\|z P^{\prime}(z)+n m \beta\right\|_{p}^{*} \leqslant \frac{n}{\|1+z\|_{p}^{*}}\|P\|_{p}^{*} .
$$

Remark 1. Similarly, if we choose $\beta$ and $\lambda_{i}=0,1,2$ suitably in Corollary 2, many known polynomial inequalities can be obtained by a fairly uniform procedure. 
Remark 2. Apparently it seems that Theorem 1 follows from a result earlier proved by Wali and Liman [16]. But the parameter of $z^{n}$ in this theorem makes it different and more interesting. The comparison between the two is also obvious by the corollaries deduced and the lemmas used in the two papers, besides the methods used to prove the various results.

2. Lemmas. For the proof of the theorem, we need the following Lemmas. The first Lemma follows from [9, Corollary 18.3]:

Lemma 1. If all the zeros of a polynomial $P(z)$ of degree $n$ lie in a circle $|z| \leqslant 1$, then all the zeros of the polynomial $B[P](z)$ also lie in $|z| \leqslant 1$.

The next Lemma we require is due to Aziz and Shah [4].

Lemma 2. If $L, M, N$ are non-negative real numbers, such that $M+N \leqslant L$, then for every real number $\alpha$

$$
\left|(L-N)+e^{i \alpha}(M+N)\right| \leqslant\left|L+e^{i \alpha} M\right| .
$$

Lemma 3. If $P \in \mathcal{P}_{n}$ and $P(z) \neq 0$ in $|z|<1$, then for $|z| \geqslant 1$,

$$
|B[P]| \leqslant\left|B\left[P^{*}\right]\right|
$$

where $B \in \mathcal{B}_{n}$ and $P^{*}(z):=z^{n} \overline{P\left(\frac{1}{z}\right)}$.

The above lemma is due to Shah and Liman [14, Lemma 2.2].

Lemma 4. If $P \in \mathcal{P}_{n}$ and $P^{*}(z):=z^{n} \overline{P\left(\frac{1}{\bar{z}}\right)}$, then we have

$$
\left|B\left[P^{*}\right](R z)\right|=\left|B\left(\left[P^{*}\right](R z)\right)^{*}\right|:=\left|\left(B\left[P^{*}\right](R z)\right)^{*}\right|, \text { for }|z|=1 .
$$

Proof. For $P \in \mathcal{P}_{n}$, we have $P^{*}(R z)=R^{n} z^{n} \overline{P\left(\frac{1}{R \bar{z}}\right)}$. This gives

$$
\begin{aligned}
& B\left[P^{*}\right](R z)=B\left[P^{*}(R z)\right]= \\
= & \lambda_{0} R^{n} z^{n} \overline{P(1 / R \bar{z})}+\lambda_{1}\left(\frac{n z}{2}\right)\left[n R^{n} z^{n-1} \overline{P(1 / R \bar{z})}-R^{n-1} z^{n-2} \overline{P^{\prime}(1 / R \bar{z})}\right]+ \\
+ & \frac{\lambda_{2}}{2 !}\left(\frac{n z}{2}\right)^{2}\left[n(n-1) R^{n} z^{n-2} \overline{P(1 / R \bar{z})}-2(n-1) R^{n-1} z^{n-3} \overline{P^{\prime}(1 / R \bar{z})}+\right. \\
& \left.+R^{n-2} z^{n-4} \overline{P^{\prime \prime}(1 / R \bar{z})}\right]=\left(\lambda_{0}+\frac{n^{2}}{2} \lambda_{1}+\frac{n^{3}(n-1)}{8} \lambda_{2}\right) R^{n} z^{n} \overline{P(1 / R \bar{z})}- \\
& -\left\{\frac{n}{2} \lambda_{1}+\frac{n^{2}(n-1)}{4} \lambda_{2}\right\} R^{n-1} z^{n-1} \overline{P^{\prime}(1 / R \bar{z})}+\frac{n^{2}}{8} \lambda_{2} R^{n-2} z^{n-2} \overline{P^{\prime \prime}(1 / R \bar{z})}
\end{aligned}
$$


Also,

$$
\begin{gathered}
\left(B\left[P^{*}\right](R z)\right)^{*}=z^{n} \overline{B\left[P^{*}(R / \bar{z})\right]}=\left(\overline{\lambda_{0}}+\frac{n^{2}}{2} \overline{\lambda_{1}}+\frac{n^{3}(n-1)}{8} \overline{\lambda_{2}}\right) R^{n} P(z / R)- \\
-\left\{\frac{n}{2} \overline{\lambda_{1}}+\frac{n^{2}(n-1)}{4} \overline{\lambda_{2}}\right\} R^{n-1} z P^{\prime}(z / R)+\frac{n^{2}}{8} \overline{\lambda_{2}} R^{n-2} z^{2} P^{\prime \prime}(z / R) . \quad \text { (11) }
\end{gathered}
$$

From (10), (11) it follows that $\left|B\left[P^{*}\right](R z)\right|=\left|\left(B\left[P^{*}\right](R z)\right)^{*}\right|$ for $|z|=1$.

Lemma 5. If $P \in \mathcal{P}_{n}$, then for every $p \geqslant 0, R \geqslant 1$ and $0 \leqslant \alpha<2 \pi$,

$$
\begin{gathered}
\int_{0}^{2 \pi} \int_{0}^{2 \pi}\left|B[P]\left(R e^{i \theta}\right)+e^{i \alpha}\left(B\left[P^{*}\right]\left(R e^{i \theta}\right)\right)^{*}\right|^{p} d \theta d \alpha \leqslant \\
\leqslant \int_{0}^{2 \pi}\left|R^{n} \wedge e^{i \alpha}+\lambda_{0}\right|^{p} d \alpha \int_{0}^{2 \pi}\left|P\left(e^{i \theta}\right)\right|^{p} d \theta,
\end{gathered}
$$

where $B$ and $\wedge$ are defined in (6).

The above Lemma is due to Rather and Shah [12, Lemma 6].

3. Proof of the Theorem 1. If $P(z)$ has a zero on $|z|=1$, then $m=\min _{|z|=1}|P(z)|=0$ and the result follows from Theorem B. We suppose that $P(z)$ has no zeros on $|z|=1$, so that $m>0$, and we have $\left|m \beta z^{n}\right|<|P(z)|$ for $|z|=1$ for any $\beta$ with $|\beta|<1$. By Rouche's theorem, the polynomial $F(z)=P(z)+\beta m z^{n}$ has all its zeros in $|z|>1$. By Lemma 3, we have

$$
|B[F](z)| \leqslant\left|B\left[F^{*}\right](z)\right| \text { for }|z| \geqslant 1,
$$

where $F^{*}(z)=z^{n} \overline{F\left(\frac{1}{\bar{z}}\right)}$. In particular, for every $R>1$ we have

$$
\left|B\left[P(R z)+\beta m R^{n} z^{n}\right]\right| \leqslant\left|B\left[P^{*}(R z)+\bar{\beta} m\right]\right| .
$$

Since $P(z)$ and $z^{n}$ are of same degree and $B$ is linear in this case,

$$
B\left[P(R z)+\beta m R^{n} z^{n}\right]=B[P](R z)+\beta m B\left[E_{n}(R z)\right],
$$

where $\left[E_{n}\right](z)=z^{n}$. Also, it can be easily verified that

$$
B\left[P^{*}(R z)+\beta m\right]=B\left[P^{*}\right](R z)+\beta m \lambda_{0}
$$


for $|z|=1$. Therefore, we have, from inequality (12),

$$
\left|B[P](R z)+m \beta B\left[E_{n}\right](R z)\right| \leqslant\left|B\left[P^{*}\right](R z)+m \bar{\beta} \lambda_{0}\right|, \quad|z|=1 .
$$

Choosing the argument of $\beta$ suitably in the left-hand side of (13) and using the triangle inequality in the right-hand side, we get from (13):

$$
|B[P](R z)|+m|\beta|\left|B\left[E_{n}\right](R z)\right| \leqslant\left|B\left[P^{*}\right](R z)\right|+m|\beta|\left|\lambda_{0}\right| .
$$

Equivalently,

$$
|B[P](R z)| \leqslant\left|B\left[P^{*}\right](R z)\right|-m|\beta|\left\{\left|B\left[E_{n}\right](R z)\right|-\left|\lambda_{0}\right|\right\} .
$$

Since $\left|B\left[E_{n}\right](R z)\right|=R^{n}|\wedge|$ for $|z|=1$, this gives

$$
\begin{aligned}
|B[P](R z)|+ & \left\{\frac{|\wedge| R^{n}-\left|\lambda_{0}\right|}{2}\right\} m|\beta| \leqslant \\
& \leqslant\left|B\left[P^{*}\right](R z)\right|-\left\{\frac{|\wedge| R^{n}-\left|\lambda_{0}\right|}{2}\right\} m|\beta| .
\end{aligned}
$$

If we take $L=\left|B\left[P^{*}\right](R z)\right|, M=|B[P](R z)|$ and $N=\left\{\frac{|\wedge| R^{n}-\left|\lambda_{0}\right|}{2}\right\} m|\beta|$, so that $M+N \leqslant L-N \leqslant L$ and we get, by using Lemma 2 , for every real $\alpha$ and $|z|=1$,

$$
\begin{aligned}
& || B\left[P^{*}\right](R z)\left|-\left\{\frac{|\wedge| R^{n}-\left|\lambda_{0}\right|}{2}\right\} m\right| \beta \mid+ \\
+ & e^{i \alpha}\left[|B[P](R z)|+\left\{\frac{|\wedge| R^{n}-\left|\lambda_{0}\right|}{2}\right\} m|\beta|\right]|\leqslant| B[P](R z)\left|+e^{i \alpha}\right| B\left[P^{*}\right](R z) \mid .
\end{aligned}
$$

This gives, for each $p>0,0 \leqslant \theta<2 \pi$,

$$
\int_{0}^{2 \pi}\left|G(\theta)+e^{i \alpha} F(\theta)\right|^{p} d \theta \leqslant \int_{0}^{2 \pi}|| B[P]\left(R e^{i \theta}\right)\left|+e^{i \alpha}\right| B\left[P^{*}\right]\left(R e^{i \theta}\right)||^{p} d \theta
$$

where

$$
F(\theta)=\left|B[P]\left(R e^{i \theta}\right)\right|+\left\{\frac{|\wedge| R^{n}-\left|\lambda_{0}\right|}{2}\right\} m|\beta|
$$

and

$$
G(\theta)=\left|B\left[P^{*}\right]\left(R e^{i \theta}\right)\right|-\left\{\frac{|\wedge| R^{n}-\left|\lambda_{0}\right|}{2}\right\} m|\beta|
$$


Integrating both sides of (16) with respect to $\alpha$ from 0 to $2 \pi$ and then using Lemma 3, we get

$$
\begin{aligned}
\int_{0}^{2 \pi} \int_{0}^{2 \pi}\left|G(\theta)+e^{i \alpha} F(\theta)\right|^{p} d \theta d \alpha \leqslant \\
\quad \leqslant \int_{0}^{2 \pi} \int_{0}^{2 \pi}|| B[P]\left(R e^{i \theta}\right)\left|+e^{i \alpha}\right| B\left[P^{*}\right]\left(R e^{i \theta}\right)||^{p} d \theta d \alpha= \\
\quad=\int_{0}^{2 \pi} \int_{0}^{2 \pi}|| B[P]\left(R e^{i \theta}\right)\left|+e^{i \alpha}\right|\left(B\left[P^{*}\right]\left(R e^{i \theta}\right)\right)^{*}||^{p} d \theta d \alpha .
\end{aligned}
$$

Using Lemma 5, this gives for every real $\alpha$ and $0 \leqslant \theta<2 \pi$,

$$
\int_{0}^{2 \pi} \int_{0}^{2 \pi}\left|G(\theta)+e^{i \alpha} F(\theta)\right|^{p} d \theta d \alpha \leqslant \int_{0}^{2 \pi}\left|R^{n} \wedge e^{i \alpha}+\lambda_{0}\right|^{p} d \alpha \int_{0}^{2 \pi}\left|P\left(e^{i \theta}\right)\right|^{p} d \theta .
$$

Now, we know that for every real $\alpha$ and $r \geqslant 1$

$$
\left|r+e^{i \alpha}\right| \geqslant\left|1+e^{i \alpha}\right|
$$

This implies, for each $p>0$,

$$
\int_{0}^{2 \pi}\left|r+e^{i \alpha}\right|^{p} d \alpha \geqslant \int_{0}^{2 \pi}\left|1+e^{i \alpha}\right|^{p} d \alpha
$$

Now, if $F(\theta) \neq 0$, then from (15) for $r=\frac{|G(\theta)|}{|F \theta|} \geqslant 1$, we have, by using (18),

$$
\begin{aligned}
& \int_{0}^{2 \pi}\left|G(\theta)+e^{i \alpha} F(\theta)\right|^{p} d \alpha=|F(\theta)|^{p} \int_{0}^{2 \pi}\left|\frac{G(\theta)}{F(\theta)}+e^{i \alpha}\right|^{p} d \alpha= \\
& =|F(\theta)|^{p} \int_{0}^{2 \pi}|| \frac{G(\theta)}{F(\theta)}\left|+e^{i \alpha}\right|^{p} d \alpha \geqslant|F(\theta)|^{p} \int_{0}^{2 \pi}\left|1+e^{i \alpha}\right|^{p} d \alpha= \\
& =\left|B[P]\left(R e^{i \theta}\right)+\left\{\frac{R^{n}|\wedge|-\left|\lambda_{0}\right|}{2}\right\} m\right| \beta||_{0}^{p}\left|1+e^{i \alpha}\right|^{p} d \alpha .
\end{aligned}
$$


Clearly, Inequality (19) is trivial in the case $F(\theta)=0$. Now, integrating the two sides of (19) with respect to $\theta$ and then using (17), we get

$$
\begin{aligned}
\int_{0}^{2 \pi}\left|B[P]\left(R e^{i \theta}\right)+\left\{\frac{R^{n}|\wedge|-\left|\lambda_{0}\right|}{2}\right\} m\right| \beta||^{p} d \theta \int_{0}^{2 \pi}\left|1+e^{i \alpha}\right|^{p} d \alpha \leqslant \\
\leqslant \int_{0}^{2 \pi}\left|R^{n} \wedge e^{i \alpha}+\lambda_{0}\right|^{p} d \alpha \int_{0}^{2 \pi}\left|P\left(e^{i \theta}\right)\right|^{p} d \theta,
\end{aligned}
$$

for every $\beta$ with $|\beta|<1, R>1$, and $p>0$, where $\wedge$ is defined by (6), $\alpha$ is real, and $0 \leqslant \theta<2 \pi$. This, in particular, gives

$$
\begin{aligned}
\int_{0}^{2 \pi} \mid B[P]\left(R e^{i \theta}\right)+\left\{\frac{R^{n}|\wedge|-\left|\lambda_{0}\right|}{2}\right\} & \left.m|\beta|\right|^{p} d \theta \leqslant \\
& \leqslant \frac{\int_{0}^{2 \pi}\left|R^{n} \wedge e^{i \alpha}+\lambda_{0}\right|^{p} d \alpha}{\int_{0}^{2 \pi}\left|1+e^{i \alpha}\right| p d \alpha} \int_{0}^{2 \pi}\left|P\left(e^{i \theta}\right)\right|^{p} d \theta .
\end{aligned}
$$

And this, in turn, gives the desired result.

For the equality case, note that for the polynomial $P(z)=z^{n}+1$, $m=0$, and $\left\|1+z^{n}\right\|_{p}=\|1+z\|_{p}$; so that

$$
\begin{gathered}
\left\|B[P](R .)+\left(R^{n} \Lambda z^{n}-\left|\lambda_{0}\right|\right) m|\beta|\right\|_{p}= \\
=\left\|\left(\lambda_{0}+\lambda_{1} \frac{n^{2}}{2}+\lambda_{2} \frac{n^{2}(n-1)}{8}\right) R^{n} z^{n}+\lambda_{0}\right\|_{p}= \\
\quad=\left\|R^{n} \Lambda z^{n}+\lambda_{0}\right\|_{p}=\frac{\left\|R^{n} \Lambda z^{n}+\lambda_{0}\right\|_{p}}{\|1+z\|_{p}}\|P\|_{p} .
\end{gathered}
$$

\section{References}

[1] V. V. Arestov, On integral inequalities for trigonometric polynomials and their derivatives., Math. USSR-Izv., vol. 18, pp. 1-17.

[2] N. C. Ankeny and T. J. Rivlin, On a Theorem of S. Bernstein, Pacific. J. Math., 1955, vol. 5, 849-852.

[3] A. Aziz and Q. M. Dawood, Inequalities for a polynomial and its derivative, J. Approx. Theory., 1988. vol. 53 , pp. 155-162. 
[4] Abdul Aziz and W. M. Shah, $L^{p}$ inequalities for polynomials with restricted zeros, Glasnik. Matematički, 1997, vol. 52 , pp. 247-258.

[5] S. Bernstein, Sur la limitation des dérivées des polynomes C. R. Acad. Sci. Paris., 1930, vol. 190, pp. 338-340.

[6] R. P. Boas Jr and Q. I. Rahman, Lp inequalities for polynomials and entire functions, Arch. Ration. Mech. Anal., 1962, vol.11, pp. 34-39.

[7] N. G. de Bruijn, Inequalities Concerning Polynomials in the Complex Domain, Nederal. Akad. Wetensch. Proc., 1947, vol.50, pp. 1265-127.

[8] P. D. Lax, Proof of a conjecture of P. Erdös on the derivative of a polynomial, Bull. Amer. Math. Soc., 1944, (N. S.) vol. 50, pp. 509-513.

[9] M. Marden, Geometry of polynomials, 2nd ed., Mathematical Surveys No.3, Amer. Math. Soc, Providence, RI, 1966.

[10] Q. I. Rahman, Functions of exponential type, Trans. Amer. Math. Soc,. 1969, vol .135, pp. $295-300$.

[11] Q. I. Rahman and G. Schmeisser, Analytic Theory of Polynomials, Oxford University Press, New York, 2002.

[12] N. A. Rather and M. A. Shah, On an operator preserving $L_{p}$ inequalities between Polynomials, J. Math. Appl., 2013, vol 399 , pp. 422-432.

DOI: https://doi.org/10.1016/j.jmaa.2012.10.031.

[13] N. A. Rather, Suhail Gulzar and K. A. Thakur, Some integral mean estimates for polynomials, New Zealand. J. Math., 2014, vol 44, pp. 83-91.

[14] W. M. Shah, A. Liman, An operator preserving inequalities between polynomials, J. Inequal. Pure Appl. Math., 2008, vol. 9, pp. 1-12.

[15] W. M. Shah, A. Liman, Integral estimates for the family of B-operators. Oper. Matrices., 2011, vol. 5 pp. $79-87$.

DOI: https://doi.org/10.7153/oam-05-04.

[16] S. L. Wali, A. Liman, Integral estimates for a class $B_{n}$-operators. Probl. Stud. Univ. Babeës-Bolyai. Math., 2018, vol 63, No. 2, pp. 175-188.

DOI: https://doi.org/10.24193/subbmath.2018.2.02.

[17] S. L. Wali, W. M. Shah and A. Liman, Inequalities concerning B-operators. Probl. Anal. Issues Anal., 2016, vol.5, no. 2, pp. 55-72.

DOI: https://doi.org/10.15393/j3.art.2016.3250.

Received March 22, 2020.

In revised form, September 15, 2020.

Accepted October 14, 2020.

Published online November 15, 2020. 
Central University of Kashmir,

Ganderbal - 191201, Jammu and Kashmir, India

E-mail:shahlw@yahoo.co.in 